\title{
Family-Centered Management of Sensory Challenges of Children With Autism: Single-Case Experimental Design
}

\author{
Kim Bulkeley, Anita Bundy, Jacqueline Roberts, Stewart Einfeld
}

\section{MeSH TERMS}

- activities of daily living

- autistic disorder

- child behavior

- developmental disabilities

- parenting

- sensation disorders
We explored the effectiveness of a sensory-based, family-centered coaching approach to changing problematic routines for young children with autism. Three mothers of young children with autism, atypical sensory processing, and global developmental delay each participated in a single-case experimental ABA design study. Mothers selected a problematic daily routine linked to sensory challenges as the focus of four intervention sessions provided in the home. Changes in mothers' perceptions of the children's behavior were the primary outcome, measured daily on a visual analog scale. Visual and descriptive analyses were undertaken. The sensory-based, family-centered coaching approach showed promise for changing sensory-related problem behaviors of young children with autism, but the degree and maintenance of the intervention effect varied among participants.

Bulkeley, K., Bundy, A., Roberts, J., \& Einfeld, S. (2016). Family-centered management of sensory challenges of children with autism: Single-case experimental design. American Journal of Occupational Therapy, 70, 7005220040. http://dx.doi.org/10.5014/ajot.2016.017822

Kim Bulkeley, BAppSc(0T), is Associate Lecturer, Faculty of Health Sciences, University of Sydney, Sydney, New South Wales, Australia; kim.bulkeley@sydney.edu.au

Anita Bundy, ScD, OT, FAOTA, is Professor, Department of Occupational Therapy, Colorado State University, Fort Collins, and Faculty of Health Sciences, University of Sydney, Sydney, New South Wales, Australia.

Jacqueline Roberts, BA (Hons), DipTeach, B Applied Science (Speech Pathology), PhD, is Professor of Autism, Autism Centre of Excellence, School of Education and Professional Studies, Griffith University, Brisbane, Queensland, Australia.

Stewart Einfeld, MD, GradCertMan (CSU) DCH (RCP\&S) FRANZCP, is Chair of Mental Health and Senior Scientist, Faculty of Health Sciences, Brain and Mind Centre, University of Sydney, Sydney, New South Wales, Australia.
U nusual responses to sensory features of the environment are a pervasive issue for young children with autism spectrum disorder (ASD), and as many as 95\% of 3- to 6-yr-olds with ASD experience atypical sensory processing (Tomchek \& Dunn, 2007). The significance of problematic sensory behaviors has been acknowledged by their inclusion in the recent Diagnostic and Statistical Manual of Mental Disorders (5th ed.; American Psychiatric Association, 2013) criteria for ASD as "hyper- or hyporeactivity to sensory input or unusual interests in sensory aspects of the environment" (Black \& Grant, 2014, p. 42). Atypical sensory processing has been linked to behaviors that interrupt family routines (Bagby, Dickie, \& Baranek, 2012; Schaaf, Toth-Cohen, Johnson, Outten, \& Benevides, 2011); adverse reactions to everyday sounds (Ben-Sasson et al., 2009; Koegel, Openden, \& Koegel, 2004); mealtime problems and restricted diet (Cermak, Curtin, \& Bandini, 2010); and dysregulated, unruly behavior (O'Donnell, Deitz, Kartin, Nalty, \& Dawson, 2012). Professionals using a sensory framework provide expertise in this area (Ashburner, Rodger, Ziviani, \& Jones, 2014), leading to greater involvement in remedial and adaptive interventions for children with autism.

A recent survey of occupational therapists working with children with ASD in Australia found that $65 \%$ used sensory approaches. These approaches include managing sensory processing differences using explanation; adapting tasks, routines, and environments; improving self-regulation; and using remedial approaches such as sensory integration. A similar proportion (67\%) used familycentered approaches (Ashburner, Rodger, Ziviani, \& Hinder, 2014). Familycentered practices prioritize partnerships with families, mobilizing strengths to generate solutions to family-identified goals; they are considered best practice in early intervention services (Dunn, Cox, Foster, Mische-Lawson, \& Tanquary, 2012). 
Parent-directed, family-centered interventions for children with autism are emerging, with questions arising concerning the essential components of successful interventions and the optimal amount of intervention required (Vismara, Colombi, \& Rogers, 2009). Researchers have investigated family-centered, sensory-based interventions in two studies with children with ASD. Dunstan and Griffiths (2008) completed a case study focused on managing sensory issues in daily routines with a 4-yr-old child with ASD. Dunn and colleagues (2012) applied a pretest-posttest design to individualized coaching based on sensory approaches with 20 families who nominated the intervention targets. Increased participation in daily routines and improved parent confidence were reported in both studies, providing preliminary support for combined family-centered and sensory-based approaches.

In this article, we explore the hypothesis that mothers will better manage their children's behavior challenges in the context of daily routines after a family-centered coaching intervention using sensory-based strategies (Anzalone \& Williamson, 2000; Dunn et al., 2012). This study differs from previous work because it used a singlecase experimental design (SCED) and repeated measurement using a visual analog scale (VAS).

\section{Method}

The study was approved by the Human Research Ethics Committee at the Children's Hospital Westmead (No. 08/CHW/58) and endorsed by the University of Sydney Human Research Ethics Committee. This study is part of a larger $\mathrm{ABAC}-\mathrm{A} / \mathrm{ACAB}-\mathrm{A}$ study in which participants were randomly allocated to order of intervention. Here, we report on the ABA phases of the 3 participants who received family-centered coaching based in principles of sensory integration theory as their initial intervention.

\section{Participants}

Participants were recruited through two child development clinics in which staff provided study information to parents of children newly diagnosed with ASD. Families were assessed on entry to the study and included if their child was between the ages of 3 and $5 \mathrm{yr}$ and had (1) atypical sensory processing, as determined with the Infant/
Toddler Sensory Profile ${ }^{\circledR}$ (ITSP; Dunn, 2002); (2) a behavior, nominated by the family, that interfered with daily routines and was logically linked with sensory challenges (judged by the therapist); (3) ASD, based on scores on the Autism Diagnostic Observation Schedule (Lord, Rutter, \& Le Couteur, 1995), administered by a psychologist trained to research reliability level; and (4) global developmental delay confirmed by clinic reports provided by parents.

Families did not discontinue other interventions but agreed that the target behavior would not be addressed by other service providers during the study period. Thirteen families were recruited. Seven families met inclusion criteria, but 2 discontinued participation because of family circumstances unrelated to the study. This article focuses on the 3 participants allocated to the sensory-based intervention. The mothers were a relatively well-educated group in a narrow age band of 33-38 yr (mean $[M]=$ $35.3 \mathrm{yr}$, standard deviation $[S D]=2.5$ ), predominantly from a non-English-speaking background. Children ranged in age from 48 to $57 \mathrm{mo}(M=52.3 \mathrm{mo}, S D=$ 4.5) on entry to the study (Table 1). All mothers spoke conversational English.

\section{Instruments}

VASs are a simple, individualized data collection option, and a promising body of work in educational settings has supported the sensitivity (e.g., Chafouleas, Sanetti, Kilgus, \& Maggin, 2012), reliability, concurrent validity, and generalizability of a single-item VAS (e.g., Chafouleas et al., 2010). An individualized VAS was developed for each participant and programmed into an electronic handheld device (Palm Z22; Palm, Inc., Sunnyvale, CA) using the Experience Sampling Program (Barrett \& Barrett, 2005). The VAS was anchored at both ends by statements relevant to the target behavior; specific criteria describing the target behavior were displayed each time the VAS was opened. The language and phrasing of the descriptors were developed collaboratively with the mothers to ensure their focus on the same behavior over time.

Mothers received training with the Palm and were encouraged to find a suitable time to rate their perceptions of their child's behavior each day. They moved a slider

Table 1. Summary of Participants' Characteristics

\begin{tabular}{lclll}
\hline Participant & Mother's Age, Yr & Cultural Background & Child Age, Mo & Target Behavior \\
\hline 1 & 33 & Vietnamese & 57 (female) & Reaction to hair dryer noise \\
2 & 38 & Indian & 52 (male) & Rigid eating behaviors \\
3 & 35 & Australian-Asian & 48 (male) & Reckless, dangerous behavior \\
\hline
\end{tabular}


along the VAS to reflect their perceptions of the behavior over the whole day, generating a score out of 100 (Figure 1).

The ITSP (Dunn, 2002) was used to confirm atypical sensory processing. The ITSP (for ages 7-36 mo) was used because, although participants were older than the specified age range, examination of items included in other instruments revealed that the ITSP was the best tool to capture the constructs under study. The ITSP includes 48 items rated by carers on a 5-point scale; typical performance falls within $1 S D$ of the mean. Although the ITSP's psychometric properties have yet to be confirmed, with the strongest evidence for construct validity and mixed results for reliability (Dunn, 2002), it is the preferred option for assessing sensory processing in young children (Eeles et al., 2013).

Scores above the cutoff on Module 1 of the ADOS were the criterion for autism. Module 1 has evidence for excellent interrater reliability, internal consistency, and test-retest reliability. It is considered the gold standard for autism diagnosis when administered by a trained assessor (Lord et al., 2000).

\section{Experimental Design and Procedures}

A SCED was applied as a non-concurrent, ABA protocol (Hayes, 1981; Watson \& Workman, 1982). A nonconcurrent SCED was considered appropriate because participants were in independent locations and shared no common context or history.

The first author (Bulkeley) worked with each family to identify behaviors that interfered with family routines that could be linked to sensory processing challenges and establish the family's focus for the intervention. The mother was the focus of the coaching intervention, with sessions scheduled in the home at a mutually convenient time. The initial baseline was set at a minimum of $2 \mathrm{wk}$ to establish a pattern of ratings that reflected daily life as usual. Baseline varied in length across participants to accommodate family circumstances (e.g., illness; moving

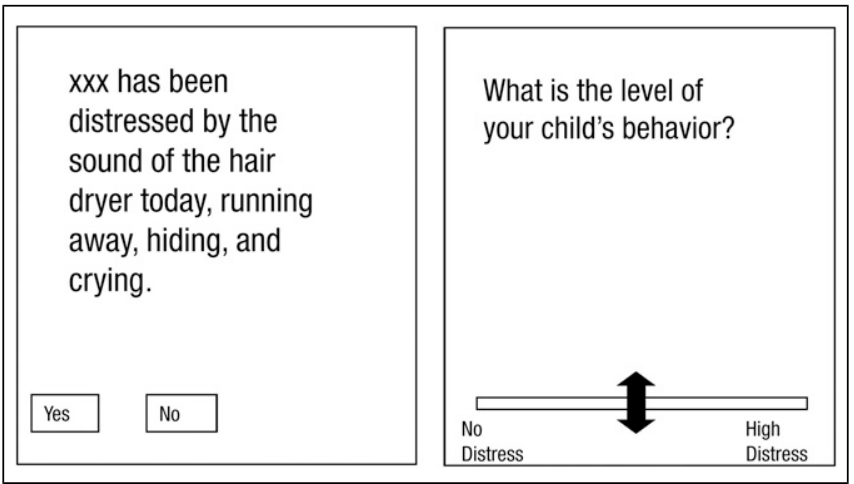

Figure 1. Sample VAS rating scale screen images from Palm Z22. Note. VAS $=$ visual analog scale. house). The initial baseline commenced after identification of the target behavior. The mother was asked to record her perception of the child's behavior daily on the VAS at a convenient time.

The intervention phase commenced with the first of four 1-hr intervention sessions. The sessions were meant to occur once a week, but family schedules resulted in some occurring more than $1 \mathrm{wk}$ apart. The therapist made an effort to visit the families' homes at times when the target behaviors were likely to occur so that she could observe the behaviors in an unstructured way. The intervention occurred in the relevant area of the home, most often the living room, and consisted of discussion, reframing, and joint problem solving. It often included demonstration coupled with mothers' trialing proposed strategies and used handouts such as sensory stories and social stories. Sessions were audio recorded to allow for independent assessment of fidelity. The second baseline commenced $1 \mathrm{wk}$ after the final intervention session. Mothers were encouraged to continue with routines and strategies that were developed during the intervention but did not receive any support or coaching during this time.

Intervention drew on sensory integration theory (Bundy, Lane, \& Murray, 2002). Five components built on an approach adapted from Anzalone and Williamson (2000) were considered as follows:

1. Observing and reframing sensory processing challenges in context (e.g., the child has an extreme reaction to the vacuum cleaner because of auditory overresponsiveness), with the mother and the therapist together observing and discussing the behavior and developing management strategies

2. Modifying the environment to reduce contributing sensory input (e.g., close doors to place a barrier between the child and the vacuum cleaner)

3. Modifying the activity to minimize unpleasant sensations (e.g., vacuum when the child is not home)

4. Managing the activity (e.g., use visuals to prepare for vacuuming; use calming sensory activities)

5. Promoting agency in the child in response to sensory challenges (e.g., teach the child to turn on a favorite TV show during vacuuming).

For Participant 1, strategies included increasing understanding of the child's auditory sensitivity, closing two doors between the child and the hair dryer, and encouraging the child to cuddle her blanket and put on her favorite TV show at high volume during hair drying. For Participant 2, strategies included increasing understanding of food textural tolerance, providing opportunities to engage in calming movement activities before eating, and developing independent use of a spoon. For 
Participant 3, strategies included reframing reckless behavior by discussing potential motivators and increasing the opportunities for acceptable and independently accessible alternative activities.

\section{Fidelity to Treatment}

An intervention fidelity checklist (available on request from the first author) was completed by an independent rater, unfamiliar with the study, who rated the presence of the five components of the sensory intervention outlined in the procedures. The rater completed the checklist after listening to two randomly selected audio recordings from a pool of 12 (4 per child).

\section{Data Analysis}

Significant autocorrelation was found in the data for all participants ( $r s=.23-.66, M=0.46, S D=0.21, p<$ $.05)$, violating the assumption of normal distribution and rendering trend lines unreliable (Kazdin, 2011). Therefore, visual analysis was the primary means of examining the data, supported by descriptive analysis using median and range of VAS ratings. The median initial baseline score was used as a more robust indicator of within-phase level than a mean with variable data. Data points would be expected to fall evenly above and below the initial baseline median line if the data were similar in other phases. A greater proportion of data points below the median indicates a positive deviation from the initial baseline pattern of ratings.

\section{Results}

The overall results indicate that a brief family-centered coaching intervention using a sensory-based framework showed promise in reducing the impact of target behaviors. When phases were compared using the initial baseline median score as an indicator of the presenting level of behavior, all participants showed a greater proportion of data points below the median initial baseline score during the intervention and second baseline phases. This result indicates some degree of positive intervention effect. The descriptive summary scores (Table 2) reveal a reduced range of ratings in the second baseline phase and a reduction in median scores after the introduction of the intervention for all participants.

Participant 1 (Figure 2) sought to reduce her daughter's distress in response to hair dryer noise. In the intervention phase, $92 \%$ of data points were below the initial baseline median; at the second baseline, $100 \%$ of points were below the initial baseline median. The reduction is also evident on visual analysis, which is seen in
Table 2. VAS Behavior Ratings Descriptive Data

\begin{tabular}{lccc}
\hline & \multicolumn{3}{c}{ Participant } \\
\cline { 2 - 4 } Phase & 1 & 2 & 3 \\
\hline Initial baseline & & & \\
Mdn & 90 & 68.5 & 9 \\
$n$ & 15 & 40 & 28 \\
$\quad$ Range & $35-100$ & $39-94$ & $1-100$ \\
Intervention & & & \\
Mdn & 72 & 58 & 2 \\
$n$ & 26 & 53 & 28 \\
Range & $16-100$ & $9-66$ & $1-100$ \\
2nd baseline & & & \\
$\quad$ Mdn & 59 & 59 & 2 \\
$n$ & 13 & 13 & 13 \\
Range & $31-82$ & $39-75$ & $1-84$ \\
\hline
\end{tabular}

Note. $M d n=$ median; $n=$ number of data points; range = actual VAS scores; VAS $=$ visual analog scale.

the final four data points at baseline but persists as a predominantly downward trend in the intervention phase, punctuated by occasional high-level ratings.

Participant 2 (Figure 2) sought to reduce her son's rigid eating behaviors (e.g., eating only soft-textured food items and scraping biscuits with his fingernails before eating). The initial baseline was extended because of a delay in the family's commencing the intervention. The baseline shows variability, with a wide range of ratings and an upward trend at the end of the phase. In the intervention phase, $100 \%$ of data points fell below the baseline median; $85 \%$ fell below in the second baseline. This result demonstrates a reduction in the problematic mealtime behaviors during intervention, followed by a slight increase during the second baseline, but at a lower level than at the initial baseline. The pattern of reduced variability during the intervention and second baseline phase is a positive response to the intervention, but the variability at the end of the second baseline phase suggests a lack of maintenance of the full intervention effect.

Participant 3 (Figure 2) reported on her son's reckless and dangerous behaviors, for example, lying along the top of the flat-screen television and repeatedly banging cupboard doors. The initial baseline revealed extremely variable behavior, with a scattering of scores at the bottom of the scale, interspersed with midrange and high VAS ratings of behavior trending up at the end of the phase. The intervention phase yielded high and extremely variable behavior in the first half, with consecutive low ratings in the second half except for three high ratings toward the end of the phase. Participant 3 showed a smaller degree of reduction in behavior than the other participants, with $57 \%$ of data points below the initial baseline median during intervention. A modest decrease in behavior was 


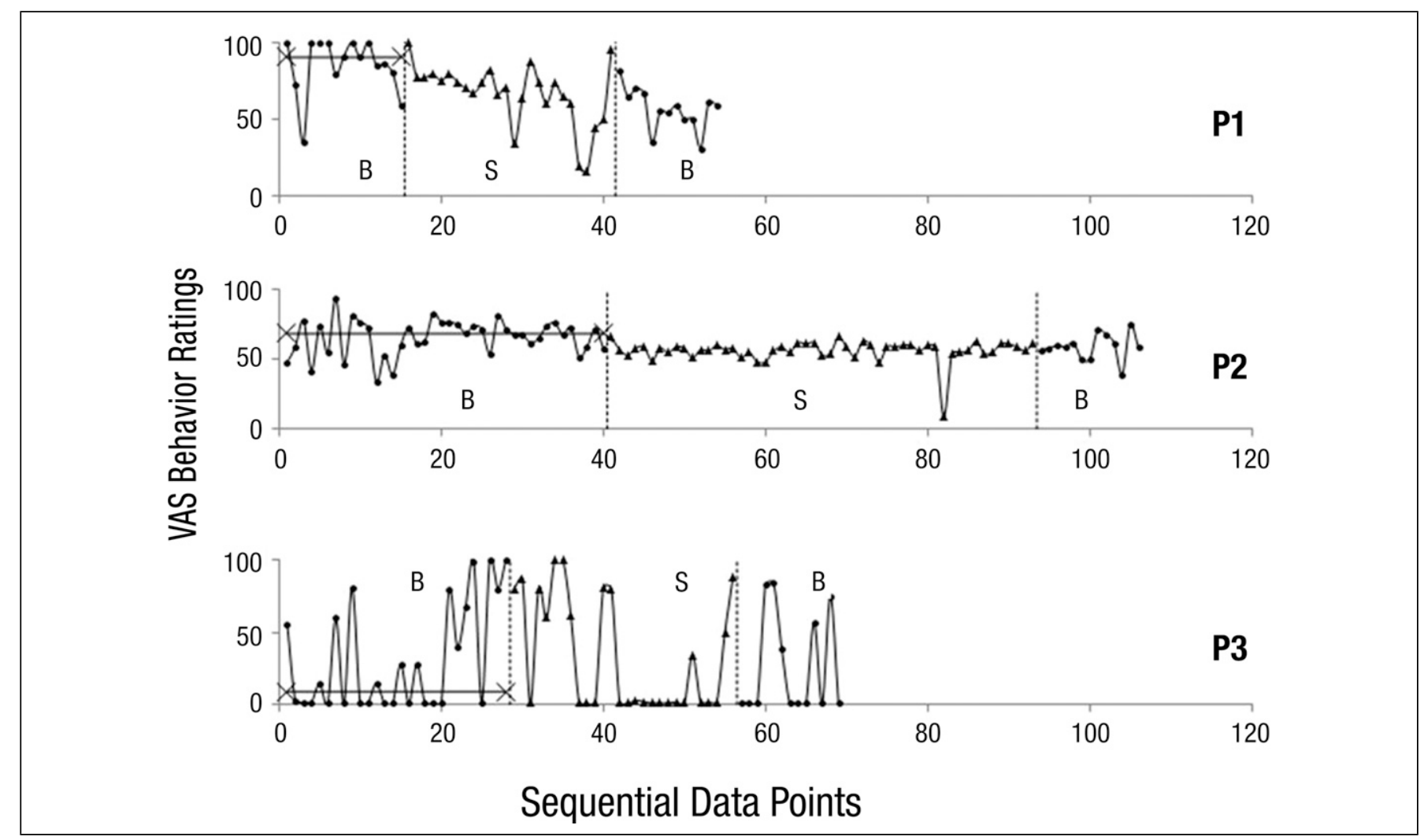

Figure 2. VAS behavior ratings.

Note. Horizontal line is initial baseline. $\mathrm{B}=$ baseline; P1-P3 = Participants $1-3 ; \mathrm{S}=$ sensory intervention; VAS = visual analog scale.

demonstrated by the greater proportion of data points below the initial baseline median (62\%) in the second baseline phase, distinguishing this phase from initial baseline patterns. However, there was still a great deal of behavior variability and consistently high levels of problematic behavior at the end of the second baseline, suggesting a reversion toward the initial baseline data patterns.

\section{Discussion}

The sensory-based, family-centered coaching approach showed promise for changing sensory-related problem behaviors of young children with autism. This intervention was acceptable to all participants because it was responsive to family priorities, informed by the cultural standpoint of participants, and embedded in the home and family context. Reframing problematic behavior through a sensory lens was the first step in developing individualized interventions to increase participation in family occupations (Bagby et al., 2012) for all participants, but the differences between the nature of the presenting issues and the specific needs of different families yielded variable responses within and among participants.

Variability in behavior is characteristic of young children with autism (Hodgetts \& Hodgetts, 2007) and requires a nuanced interpretation of intervention re- sponses. Using a VAS for repeated measurement of intervention responses revealed day-to-day variability that would not have been captured using a pre- and postevaluation method alone. Ultimately, repeated measurement with large sample sizes may enable researchers to identify subgroups of children who respond differentially to interventions.

The degree of intra- and inter-participant variability in this study highlights the complexity of interpretation of data. Nonetheless, all 3 participants showed some positive response to the sensory-based intervention, but maintenance of the full impact of the intervention was evident in only 1 participant at the end of the second baseline.

The second baseline phase reflects the removal of therapist support to the mother, who had been coached to implement identified strategies. The lack of sustained intervention impact for 2 of the participants in the second baseline phase may indicate a need in some instances for more therapist support to consolidate strategies. Vismara and colleagues (2009) found that a $12 \times 1$-hr parent training intervention with young children with autism resulted in sustained improvements, noting parental behavior change occurring from the 5 th to the 6 th $\mathrm{hr}$ of intervention. Further research is required to explore the optimal amount of intervention and any parent or child characteristics that may affect it. 
The strongest intervention response was reported by the mother of Participant 1, who had difficulties managing her daughter's extreme reactions to hair dryer noise. Auditory sensitivity is widely acknowledged in young children with autism (Ben-Sasson et al., 2009), but there has been little description of intervention. Koegel and colleagues (2004) reported positive outcomes using systematic desensitization to reduce reactions to household sounds in young children with autism, but alternative approaches may be more acceptable for young children. The sensory intervention for this participant included reframing the mother's awareness of the child's negative sensory experience, modifying the environment to reduce noise, and using calming activities to prepare for and increase tolerance of hair dryer noise. These sensory techniques applied in a coaching frame showed promising results and warrant further investigation. Nonetheless, an interpretation of a decrease in the problematic behavior as a result of intervention must be tempered somewhat because the final four data points of the initial baseline also revealed a decrease. However, the pattern of behavior in the intervention phase is substantially different from that of the initial baseline.

Food selectivity and ensuing behavior struggles were the focus for Participant 2; these are common problems for children with autism and are often linked to sensory processing difficulties (Cermak et al., 2010). The intervention reframed eating difficulties with a sensory lens and noticeably reduced the variability of the behavior but only slightly reduced the level of the VAS ratings of behavior. In addition, these reductions were not sustained toward the end of the second baseline phase. The complexity of eating issues has been noted (Marquenie, Rodger, Mangohig, \& Cronin, 2011), along with a need for comprehensive approaches to eating management. This intervention resulted in some improvements for Participant 2, but a broader approach may be required to blend sensory strategies with a range of intervention frameworks (Ashburner, Rodger, Ziviani, \& Hinder, 2014).

Participant 3 had extremely good days punctuated by extremely bad days. This kind of variable behavior has been linked to sensory processing issues (O'Donnell et al., 2012). This intervention focused on reframing behavior as communicating about his sensory needs and identifying strategies to provide more adaptive sensory experiences. Strategies included going to indoor activity centers, going to the park, sitting on an inflatable ball, and squashing himself under a child-size foam lounge cushion, but the positive changes were smaller and not fully sustained. In hindsight, these strategies were focused on behavior management and were not driven as much by participation in a family routine or occupation, as recommended by Ashburner, Rogers, Ziviani, and Jones (2014). Establishing a goal focused on participation in an occupation or routine as the starting point for children with this pattern of highly variable behavior may assist in embedding sensory strategies in family routines and requires further investigation.

The children in this study represent a subset of 3- to 5-yr-olds with ASD. They are a group with high support needs, limited language, and relatively low cognitive abilities. The gender balance of 2 boys to 1 girl is in keeping with the population with autism, but the participants' cultural diversity differed from that in the majority of ASD research. Culturally safe practice is needed with families and children with ASD because of the range in beliefs about cause, impact, and consequences (Ravindran \& Myers, 2013). The intervention approach adopted in this study was responsive to family preferences and accommodated the participants' cultural diversity.

\section{Limitations and Further Research}

Variability of response to intervention was noted in this study and has previously been noted in research with young children with autism (Magiati, Moss, Charman, $\&$ Howlin, 2011). Further studies are required with a focus on a single routine or activity to understand the diversity of responses to sensory-based interventions. This study capped the number of intervention sessions at four, and future research addressing the amount of intervention required to sustain positive outcomes is needed.

The use of a VAS to record mothers' perceptions of child behavior as the primary outcome was feasible and prioritized this perspective of problematic behavior in daily routines. However, further research is required to integrate mothers' perceptions with other outcome measures to address the limitations of a single perspective of child behavior from a non-blinded participant. Potential measures could include, for example, perceptions of multiple family members and video recordings, none of which were feasible in this study.

The intervention fidelity measure included in this research focused on the components of the sensory intervention. Future studies would benefit from including components of family-centered practice and coaching strategies in the fidelity measure as fundamental features of the intervention. 


\section{Implications for Occupational Therapy Practice}

This research has the following implications for occupational therapy practice:

- Guiding parents to understand the causes of a child's problematic behaviors is important for framing individualized interventions to address challenges in daily routines.

- Use of a VAS is feasible for tracking intervention responses over time, particularly for clients for whom variability is a defining characteristic.

- A family-centered coaching approach that used sensory-based strategies to manage challenges in daily routines showed promise for changing the problem behaviors of young children with autism.

- Four intervention sessions led to changes in target behaviors, but the optimal amount required for a sustained impact is unclear.

\section{Acknowledgments}

Thank you to the families who shared their knowledge by participating in this research and to the organizations who assisted with recruitment. We also thank SPOT on DD for funding the Autism Diagnostic Observation System assessments and purchase of Palm Pilots. This study is registered with the Australia and New Zealand Clinical Trials Register (ACTRN12616000068460).

\section{References}

American Psychiatric Association. (2013). Diagnostic and statistical manual of mental disorders (5th ed.). Arlington, VA: American Psychiatric Publishing.

Anzalone, M., \& Williamson, G. (2000). Sensory processing and motor performance in autism spectrum disorders. In A. M. Wetherby \& B. M. Prizant (Eds.), Autism spectrum disorders: A transactional developmental perspective ( $p$. 143-166). Baltimore: Paul Brookes.

Ashburner, J. K., Rodger, S. A., Ziviani, J. M., \& Hinder, E. A. (2014). Optimizing participation of children with autism spectrum disorder experiencing sensory challenges: A clinical reasoning framework. Canadian Journal of Occupational Therapy/Revue Canadienne d'Ergothérapie, 81, 29-38. http://dx.doi.org/10.1177/0008417413520440

Ashburner, J., Rodger, S., Ziviani, J., \& Jones, J. (2014). Occupational therapy services for people with autism spectrum disorders: Current state of play, use of evidence and future learning priorities. Australian Occupational Therapy Journal, 61, 110-120. http://dx.doi.org/10.1111/14401630.12083

Bagby, M. S., Dickie, V. A., \& Baranek, G. T. (2012). How sensory experiences of children with and without autism affect family occupations. American Journal of Occupational Therapy, 66, 78-86. http://dx.doi.org/10.5014/ajot.2012.000604
Barrett, D. J., \& Barrett, L. F. (2005). ESP: The Experience Sampling Program. Retrieved from http://www.esp.org

Ben-Sasson, A., Hen, L., Fluss, R., Cermak, S. A., Engel-Yeger, B., \& Gal, E. (2009). A meta-analysis of sensory modulation symptoms in individuals with autism spectrum disorders. Journal of Autism and Developmental Disorders, 39, 1-11. http://dx.doi.org/10.1007/s10803-008-0593-3

Black, D. W., \& Grant, J. E. (2014). DSM-5 ${ }^{T M}$ guidebook: The essential companion to the Diagnostic and statistical manual of mental disorders (5th ed.). Washington, DC: American Psychiatric Publishing.

Bundy, A. C., Lane, S. J., \& Murray, E. A. (Eds.). (2002). Sensory integration: Theory and practice (2nd ed.). Philadelphia: F. A. Davis.

Cermak, S. A., Curtin, C., \& Bandini, L. G. (2010). Food selectivity and sensory sensitivity in children with autism spectrum disorders. Journal of the American Dietetic Association, 110, 238-246. http://dx.doi.org/10.1016/j. jada.2009.10.032

Chafouleas, S. M., Briesch, A. M., Riley-Tillman, T. C., Christ, T. J., Black, A. C., \& Kilgus, S. P. (2010). An investigation of the generalizability and dependability of Direct Behavior Rating Single Item Scales (DBR-SIS) to measure academic engagement and disruptive behavior of middle school students. Journal of School Psychology, 48, 219-246. http://dx.doi.org/10.1016/j.jsp.2010.02.001

Chafouleas, S. M., Sanetti, L. M. H., Kilgus, S. P., \& Maggin, D. M. (2012). Evaluating sensitivity to behavioral change using Direct Behavior Rating Single-Item Scales. Exceptional Children, 78, 491-505.

Dunn, W. (2002). Infant/Toddler Sensory Profile user's manual. San Antonio, TX: Psychological Corporation.

Dunn, W., Cox, J., Foster, L., Mische-Lawson, L., \& Tanquary, J. (2012). Impact of a contextual intervention on child participation and parent competence among children with autism spectrum disorders: A pretest-posttest repeated-measures design. American Journal of Occupational Therapy, 66, 520-528. http://dx.doi.org/10.5014/ ajot.2012.004119

Dunstan, E., \& Griffiths, S. (2008). Sensory strategies: Practical support to empower families. New Zealand Journal of Occupational Therapy, 55, 5-13.

Eeles, A. L., Spittle, A. J., Anderson, P. J., Brown, N., Lee, K. J., Boyd, R. N., \& Doyle, L. W. (2013). Assessments of sensory processing in infants: A systematic review. Developmental Medicine and Child Neurology, 55, 314-326. http://dx.doi.org/10.1111/j.1469-8749.2012.04434.x

Hayes, S. C. (1981). Single case experimental design and empirical clinical practice. Journal of Consulting and Clinical Psychology, 49, 193-211. http://dx.doi.org/10.1037/0022006X.49.2.193

Hodgetts, S., \& Hodgetts, W. (2007). Somatosensory stimulation interventions for children with autism: Literature review and clinical considerations. Canadian Journal of Occupational Therapy/Revue Canadienne d'Ergothérapie, 74, 393-400. http://dx.doi.org/10.2182/cjot.07.013

Kazdin, A. (2011). Single-case research designs-Methods for clinical and applied settings. New York: Oxford University Press. 
Koegel, R. L., Openden, D., \& Koegel, L. K. (2004). A systematic desensitization paradigm to treat hypersensitivity to auditory stimuli in children with autism in family contexts. Research and Practice for Persons With Severe Disabilities, 29, 122-134. http://dx.doi.org/10.2511/rpsd.29.2.122

Lord, C., Risi, S., Lambrecht, L., Cook, E. H., Jr., Leventhal, B. L., DiLavore, P. C., . . Rutter, M. (2000). The Autism Diagnostic Observation Schedule-Generic: A standard measure of social and communication deficits associated with the spectrum of autism. Journal of Autism and Developmental Disorders, 30, 205-223. http://dx.doi.org/ 10.1023/A:1005592401947

Lord, C., Rutter, M., \& Le Couteur, A. (1995). Autism Diagnostic Observation Schedule (ADOS) manual. Los Angeles: Western Psychological Services.

Magiati, I., Moss, J., Charman, T., \& Howlin, P. (2011). Patterns of change in children with autism spectrum disorders who received community based comprehensive interventions in their pre-school years: A seven year follow-up study. Research in Autism Spectrum Disorders, 5, 1016-1027. http://dx.doi.org/10.1016/j.rasd.2010.11.007

Marquenie, K., Rodger, S., Mangohig, K., \& Cronin, A. (2011). Dinnertime and bedtime routines and rituals in families with a young child with an autism spectrum disorder. Australian Occupational Therapy Journal, 58, 145-154. http://dx.doi.org/10.1111/j.1440-1630.2010.00896.x

O’Donnell, S., Deitz, J., Kartin, D., Nalty, T., \& Dawson, G. (2012). Sensory processing, problem behavior, adaptive behavior, and cognition in preschool children with autism spectrum disorders. American Journal of Occupational Therapy, 66, 586-594. http://dx.doi.org/10.5014/ ajot.2012.004168

Ravindran, N., \& Myers, B. J. (2013). Beliefs and practices regarding autism in Indian families now settled abroad: An Internet survey. Focus on Autism and Other Developmental Disabilities, 28, 44-53. http://dx.doi.org/10.1177/ 1088357612458970

Schaaf, R. C., Toth-Cohen, S., Johnson, S. L., Outten, G., \& Benevides, T. W. (2011). The everyday routines of families of children with autism: Examining the impact of sensory processing difficulties on the family. Autism, 15, 373-389. http://dx.doi.org/10.1177/1362361310386505

Tomchek, S. D., \& Dunn, W. (2007). Sensory processing in children with and without autism: A comparative study using the Short Sensory Profile. American Journal of Occupational Therapy, 61, 190-200. http://dx.doi.org/10.5014/ajot.61.2.190

Vismara, L. A., Colombi, C., \& Rogers, S. J. (2009). Can one hour per week of therapy lead to lasting changes in young children with autism? Autism, 13, 93-115. http://dx.doi. org $/ 10.1177 / 1362361307098516$

Watson, P. J., \& Workman, E. A. (1982). Response to Mansell: Further clarification of the nonconcurrent multiple baseline across-individuals designs. Journal of Behavior Therapy and Experimental Psychiatry, 13, 261-262. http://dx.doi. org/10.1016/0005-7916(82)90018-0 
Reproduced with permission of the copyright owner. Further reproduction prohibited without permission. 